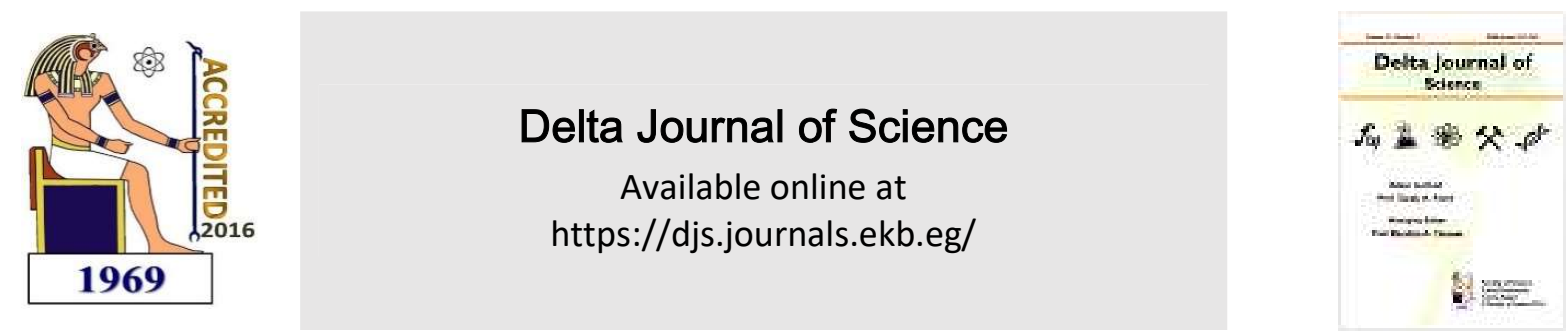

Research Article

MATHEMATICS

\title{
Two Intersection Graphs
}

\author{
M. A. Seoud* \\ Dept. of Math., Faculty of Science, Ain Shams University, Abbassia, Cairo, Egypt \\ and F. A. A. Ghouraba** \\ Ministry of Education (retired), Tanta, Egypt
}

\begin{abstract}
We give the number of edges in two intersection graphs.
Keywords : Intersection graph, Stirling numbers.

\section{Introduction}

Intersection graph theory is one of the most important topics in graph theory. There is an outstanding

calculating the number of edges of functional intersection graphs. For these numbers the reader is advised to see [2]
\end{abstract} concise book titled : "Topics in Intersection Graph Theory" by Terry A. McKee and F. R. McMorris [1], in which the most developed topics of intersection graph theory, emphasizing chordal, interval competition graphs, threshold graphs, p-intersection graphs, intersection multigraphs, pseudographs, and tolerance intersection graphs are discussed. Here we obtaied the number of edges in two intersection graphs, namely : power set intersection graphs and functional intersection graphs. Stirling numbers arise in a varity of analytic and combinatorics problems. We need stirling numbers of the second type in

\section{Power set intersection graph}

\subsection{Definition. Let $\mathrm{X}:=$} $\left\{x_{1}, x_{2}, \ldots, x_{n}\right\}$. Let $\mathrm{P}(\mathrm{X})$ be the power set of $X$, i.e. $P(X)=\{A \mid A \subseteq X\}$. The power set intersection graph is $G$ $=(\mathrm{V}, \mathrm{E})$, where $\mathrm{V}$ "corresponds to" $\mathrm{P}$ $(X)$, and two vertices in $V$ are adjacent if and only if the two corresponding subsets in $P(X)$ have a non-empty intersection.

1.2 Theorem. For a set $\mathrm{X}:=$ $\left\{x_{1}, x_{2}, \ldots, x_{n}\right\}$,the power set intersection graph $\mathbf{G}=(\mathrm{V}, \mathrm{E})$, has $|V|$, number of vertices $=2^{n}$, and $|E|$, number of edges $=\frac{1}{2}\left(4^{n}-3^{n}-2^{n}+1\right)$ 
Proof. $|V|=2^{n}$ is trivial. Now let $A \subseteq$ $X,|A|$ (number of elements of $A$ ) $=\mathbf{m}$. The degree of the vertex $v_{A}$, which "corresponds to" the set

$A=2^{n}-2^{n-m}-1$. It follows that :

$|\mathrm{E}|=\frac{1}{2} \sum_{m=1}^{n}\left(\begin{array}{c}n \\ m\end{array}\right)\left(2^{n}-2^{n-m}-1\right)$

$=$

$\frac{1}{2} \cdot 2^{n} \sum_{m=1}^{n}\left(\begin{array}{c}n \\ m\end{array}\right)-\frac{1}{2} \cdot 2^{n} \sum_{m=1}^{n}\left(\begin{array}{c}n \\ m\end{array}\right)\left(\frac{1}{2}\right)^{m}-\frac{1}{2} \sum_{m}^{n}$ $=\frac{1}{2} \cdot 2^{n}\left(2^{n}-1\right)-\frac{1}{2} \cdot 2^{n}\left(\left(1+\frac{1}{2}\right)^{n}-1\right)-$ $\frac{1}{2}\left(2^{n}-1\right)$

$=2^{2 n-1}-2^{n-1}-\frac{1}{2} \cdot 3^{n+\frac{1}{2}}$

$=\quad \frac{1}{2}\left(4^{n}-3^{n}-2^{n}\right.$

1.3 Example. The number of vertices of the power set intersection graph corresponding to the set $X$ := $\{1,2,3\}$, is $2^{3}=8$.

The number of edges = $\frac{1}{2}\left(4^{3}-3^{3}-2^{3}+1\right)=15$

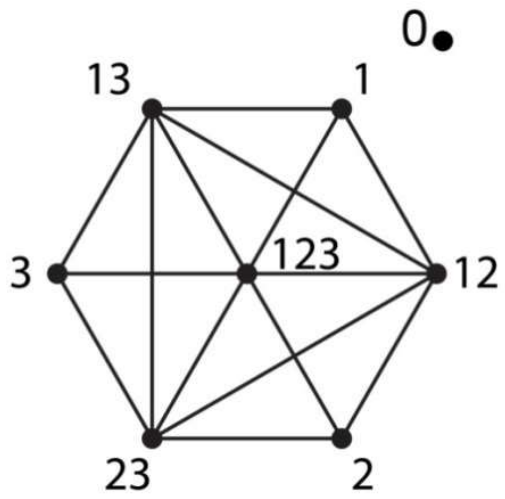

Figure 1 shows such a graph, where the vertex "123" corresponds to the subset $\{1,2,3\}$, the vertex " $0 "$ corresponds to the empty set $\varphi$. There is an edge joining the vertices "123" and "12", since the subsets $\{1,2,3\}$ and $\{1,2\}$ intersect

2. Functional intersection graph Fig.1
2.1 Definition. Let $\mathrm{L}:=\{\boldsymbol{f} \mid \boldsymbol{f}: X \rightarrow$ $Y$ \}, be the set of all functions from $X$ into $Y$. The functional intersection graph $G$ has vertices $v_{f}$ and $v_{g}$ "corresponding to" the functions $f$ and $g$ of $L$. The vertices $v_{f}$ and $v_{g}$ are adjacent if and only if range (f) and range (g) have a non-empty intersection.

2 2 Definition. Stirling number of thensecond kind $S_{r}^{m}[1]$ is equal to the number of ways of partitioning a set of $m$ elements into $r$ non-empty subsets,

$$
S_{\mathrm{r}}^{\mathrm{m}}=\frac{1}{r !} \sum_{s=1}^{r}(-1)^{r+s}\left(\begin{array}{l}
r \\
s
\end{array}\right) s^{m}
$$

2.3 Remark. The number of all surjective functions from $X$ onto $Y$, where $|X|=m,|Y|=n, n \leq m$ is equal to $n$ ! $S_{\mathbf{n}}^{\mathbf{m}}$. Consequently, for the set of all functions $f: X \rightarrow Y$ having the same range, consisting of $r$ elements, the corresponding is a complete graph consisting of

$r ! S_{r}^{m}$ vertices. We note that the number of all functions defined from $\mathrm{X}$ into $\mathrm{Y}$

$$
=\sum_{r=1}^{n}\left(\begin{array}{l}
n \\
r
\end{array}\right) r ! S_{r}^{m}=n^{m},
$$

as it is well-known.

2.4 Theorem. The number of edges of the functional intersection graph $\mathbf{G}$ corresponding to the set $L:=\{f \mid f$ : $X \rightarrow Y\}$, where $\quad \mathbf{X}:=$ $\left\{x_{1}, x_{2}, \ldots, x_{m}\right\}, Y:=\left\{y_{1}, y_{2}, \ldots, y_{n}\right\}$, is equal to

$\frac{1}{2} n^{m}\left(n^{m}-1\right) \quad-\quad \frac{1}{2} \sum_{r=1}^{n}\left(\begin{array}{l}n \\ r\end{array}\right) r !$ $S_{r}^{m}(n-r)^{m} \quad \mathrm{n} \leq \boldsymbol{m}$

$\frac{1}{2} n^{m}\left(n^{m}-1\right) \quad-\quad \frac{1}{2} \sum_{r=1}^{m}\left(\begin{array}{l}n \\ r\end{array}\right) r !$ $S_{r}^{m}(n-r)^{m} \quad \mathbf{m}<\mathbf{n}$

Proof. To explain the situation, we plot every complete subgraph of the same number of vertices in the "same plane", as follows : 


$$
\begin{array}{llll}
P_{1} \quad: & G_{1} & , & G_{2} \\
\cdots & & & , G_{n} \Rightarrow\left(\begin{array}{l}
n \\
1
\end{array}\right) \\
\text { Subgraphs } & & & \\
P_{2}: & G_{12} & , & G_{13} \\
\cdots & & & , G_{n-1 n} \Rightarrow\left(\begin{array}{l}
n \\
2
\end{array}\right)
\end{array}
$$

subgraphs

$P_{3}: G_{123}, G_{124}, \ldots$

$G_{n-2 n-1 n} \Rightarrow\left(\begin{array}{l}n \\ 3\end{array}\right)$ subgraphs

$P_{r} \quad: \quad G_{12 \ldots r} \quad, \quad \ldots$

$\Rightarrow\left(\begin{array}{l}n \\ r\end{array}\right)$ subgraphs

$P_{n-2}: G_{12 \ldots n-2}, \ldots$

$\left(\begin{array}{c}n \\ n-2\end{array}\right)=\left(\begin{array}{l}n \\ 2\end{array}\right)$ subgraphs

$P_{n-1} \quad: \quad G_{12 \ldots n-1} \quad, \quad \ldots$

$\Rightarrow\left(\begin{array}{c}n \\ n-1\end{array}\right)=\left(\begin{array}{l}n \\ 1\end{array}\right)$ subgraphs

$P_{n} \quad$ :

$\Rightarrow\left(\begin{array}{l}n \\ n\end{array}\right)=1$ subgraph

$G_{12 \ldots n}$

$\left(G_{12 \ldots r}\right.$ is the complete subgraph corresponding to all funcations $f$ : $X \rightarrow Y, X:=\left\{x_{1}, x_{2}, \ldots, x_{m}\right\}, \quad Y:=$ $\left\{y_{1}, y_{2}, \ldots, y_{n}\right\}$, their range is $\{1,2, \ldots, r\}$. This complete subgraph consists of $r ! S_{r}^{m}$ vertices, as said before )

Case $1: \mathbf{n} \leq \boldsymbol{m}$

$d_{1}$, the degree of any vertex in a subgraph in plane $P_{1}$ is given by:

$$
\begin{aligned}
& \mathrm{d}_{1}=0+\left(\left(\begin{array}{l}
\mathrm{n} \\
2
\end{array}\right)-\left(\begin{array}{c}
\mathrm{n}-1 \\
2
\end{array}\right)\right) .2 ! S_{2}^{m}+\left(\left(\begin{array}{l}
\mathrm{n} \\
3
\end{array}\right)-\right. \\
& \left.\left(\begin{array}{c}
\mathrm{n}-1 \\
3
\end{array}\right)\right) \cdot 3 ! S_{3}^{m}+\ldots \\
& \quad+\left(\left(\begin{array}{c}
\mathrm{n} \\
\mathrm{n}-1
\end{array}\right)-\left(\begin{array}{l}
\mathrm{n}-1 \\
\mathrm{n}-1
\end{array}\right)\right) \cdot(n-1) ! \\
& S_{n-1}^{m}+\left(\begin{array}{l}
\mathbf{n} \\
n
\end{array}\right) n ! S_{n}^{m}
\end{aligned}
$$

$$
\begin{aligned}
& \quad=\sum_{\alpha=2}^{n}\left(\begin{array}{l}
n \\
\alpha
\end{array}\right) \alpha ! S_{\alpha}^{m}-\sum_{\beta=2}^{n-1}\left(\begin{array}{c}
n-1 \\
\beta
\end{array}\right) \beta ! \\
& S_{\beta}^{m} \\
& =n^{m}-n-\left((n-1)^{m}-(n-1)\right) \\
& =n^{m}-(n-1)^{m}-1
\end{aligned}
$$

$d_{2}$, the degree of any vertex in a subgraph in plane $P_{2}$ is given by:

$$
\begin{aligned}
& \mathbf{d}_{2}=2-1+\left(\left(\begin{array}{l}
\mathbf{n} \\
2
\end{array}\right)-\left(\begin{array}{c}
\mathbf{n}-2 \\
2
\end{array}\right) \quad .2\right. \text { ! } \\
& S_{2}^{m}+\left(\left(\begin{array}{l}
\mathbf{n} \\
3
\end{array}\right)-\left(\begin{array}{c}
\mathbf{n}-2 \\
3
\end{array}\right)\right) \cdot 3 ! S_{3}^{m}+\ldots \\
& +\left(\left(\begin{array}{c}
n \\
n-2
\end{array}\right)-\left(\begin{array}{l}
n-2 \\
n-2
\end{array}\right)\right) \cdot(n-2) \text { ! } \\
& S_{n-2}^{m}+\left(\begin{array}{c}
n \\
n-1
\end{array}\right)(n-1) ! S_{n-1}^{m} \\
& +\left(\begin{array}{l}
\mathbf{n} \\
\mathbf{n}
\end{array}\right) n ! S_{n}^{m} \\
& =2-1+\sum_{\alpha=2}^{n}\left(\begin{array}{l}
n \\
\alpha
\end{array}\right) \alpha ! \quad S_{\alpha}^{m}- \\
& \sum_{\beta=2}^{n-2}\left(\begin{array}{c}
n-2 \\
\beta
\end{array}\right) \beta ! S_{\beta}^{m} \\
& =2-1+n^{m}-n-\left((n-2)^{m}-(n-\right. \\
& \text { 2)) } \\
& =n^{m}-(n-2)^{m-1}
\end{aligned}
$$

$d_{r}$, the degree of any vertex in a subgraph in plane $P_{r}$ is given by:

$$
\begin{aligned}
& \mathbf{d}_{\mathbf{r}}=\mathbf{r}-\mathbf{1}+\left(\left(\begin{array}{l}
\mathbf{n} \\
2
\end{array}\right)-\left(\begin{array}{c}
\mathbf{n}-\mathbf{r} \\
2
\end{array}\right) \quad .2\right. \text { ! } \\
& S_{2}^{m}+\left(\left(\begin{array}{c}
\mathbf{n} \\
3
\end{array}\right)-\left(\begin{array}{c}
\mathbf{n}-\mathbf{r} \\
3
\end{array}\right)\right) .3 ! S_{3}^{m}+\ldots \\
& +\left(\left(\begin{array}{c}
\mathbf{n} \\
\mathbf{n}-\mathbf{r}
\end{array}\right)-\left(\begin{array}{l}
\mathbf{n}-\mathbf{r} \\
\mathbf{n}-\mathbf{r}
\end{array}\right)\right) \cdot(\boldsymbol{n}-\boldsymbol{r}) ! S_{n-r}^{m} \\
& +\quad\left(\begin{array}{c}
\mathbf{n} \\
\mathbf{n}-\mathbf{r}+\mathbf{1}
\end{array}\right)(\boldsymbol{n}-\boldsymbol{r}+\mathbf{1}) \text { ! } \\
& S_{n-r+1}^{m}+\ldots+\left(\begin{array}{c}
\mathrm{n} \\
\mathrm{n}
\end{array}\right) n ! S_{n}^{m} \\
& d_{r}=r-1+\sum_{\alpha=2}^{n}\left(\begin{array}{l}
n \\
\alpha
\end{array}\right) \alpha ! \quad S_{\alpha}^{m}- \\
& \sum_{\beta=2}^{n-r}\left(\begin{array}{c}
n-r \\
\beta
\end{array}\right) \beta ! S_{\beta}^{m} \\
& =r-1+n^{m}-\mathbf{n}-((n-r))^{m}-(n- \\
& \text { r)) } \\
& =n^{m}-(n-r)^{m}-1, \quad n \leq m
\end{aligned}
$$

Now the number of all vertices in plane $P_{r}$ is given by : 


$$
\mathbf{N}_{\mathbf{r}}=\left(\begin{array}{c}
\mathbf{n} \\
\mathbf{r}
\end{array}\right) \boldsymbol{r} ! \boldsymbol{S}_{r}^{\boldsymbol{m}}
$$

hence $|E|$, the number of edges of the graph $\mathbf{G}$ is given by :

$$
\begin{aligned}
& |E|=\frac{1}{2} \sum_{r=1}^{n} N_{r} \cdot d_{r} \\
& =\frac{1}{2} \sum_{r=1}^{n}\left(\begin{array}{l}
\mathbf{n} \\
\mathbf{r}
\end{array}\right) r ! S_{r}^{m}\left(\left(n^{m}-1\right)-\right. \\
& \left.(\boldsymbol{n}-\boldsymbol{r})^{\boldsymbol{m}}\right) \\
& =\frac{1}{2} \quad\left(n^{m}-1\right) \sum_{r=1}^{n}\left(\begin{array}{l}
\mathbf{n} \\
\mathbf{r}
\end{array}\right) r ! S_{r}^{m}- \\
& \frac{1}{2} \sum_{r=1}^{n}(n-r)^{m}\left(\begin{array}{l}
\mathbf{n} \\
r
\end{array}\right) r ! S_{r}^{m} \\
& =\frac{1}{2} \quad n^{m}\left(n^{m}-1\right)- \\
& \frac{1}{2} \sum_{r=1}^{n}\left(\begin{array}{l}
\mathbf{n} \\
\mathbf{r}
\end{array}\right) r ! S_{r}^{m}(n-r)^{m}, \quad n \leq m
\end{aligned}
$$

Case 2: $\mathbf{m}<\mathbf{n}$.

Here some modifications have to be done. The number of all functions defined from $\mathrm{X}$ into $\mathrm{Y}$ is given as in case 1 by

$$
\sum_{r=1}^{m}\left(\begin{array}{l}
\mathbf{n} \\
\mathbf{r}
\end{array}\right) r ! S_{r}^{m}=n^{m}
$$

The complete subgraphs in planes $\mathbf{P}_{\mathbf{m - 2}}, \mathbf{P}_{\mathbf{m - 1}}, \mathbf{P}_{\mathbf{m}}$ are indicated as follows :

$$
\begin{aligned}
& P_{m-2}: \quad G_{12 \ldots m-2} \quad, \quad \ldots \\
& \Rightarrow\left(\begin{array}{c}
n \\
m-2
\end{array}\right) \text { subgraphs } \\
& P_{m-1} \quad: \quad G_{12 \ldots m-1} \quad, \quad \ldots \\
& \Rightarrow\left(\begin{array}{c}
n \\
m-1
\end{array}\right) \text { subgraphs } \\
& P_{m} \quad: \quad G_{12 \ldots m} \quad, \quad \ldots \\
& \Rightarrow\left(\begin{array}{c}
n \\
m
\end{array}\right) \text { subgraphs }
\end{aligned}
$$

Now $d_{1}$, the degree of any vertex in a subgraph in plane $P_{1}$ is given by:

$$
\begin{aligned}
& \mathrm{d}_{1}=\mathbf{0}+\left(\left(\begin{array}{c}
\mathbf{n} \\
2
\end{array}\right)-\left(\begin{array}{c}
\mathrm{n}-\mathbf{1} \\
2
\end{array}\right)\right) 2 ! S_{2}^{m}+\left(\left(\begin{array}{l}
\mathbf{n} \\
3
\end{array}\right)-\right. \\
& \left.\left(\begin{array}{c}
\mathrm{n}-1 \\
3
\end{array}\right)\right) 3 ! S_{3}^{m}+\ldots \\
& \quad+\left(\left(\begin{array}{c}
\mathrm{n} \\
\mathbf{m}-1
\end{array}\right)-\left(\begin{array}{c}
\mathbf{n}-\mathbf{1} \\
\mathbf{m}-1
\end{array}\right)\right)(m-1) ! \\
& S_{m-1}^{m} \\
& \quad+\left(\left(\begin{array}{c}
\mathbf{n} \\
\mathbf{m}
\end{array}\right)-\left(\begin{array}{c}
\mathbf{n}-\mathbf{1} \\
\mathbf{m}
\end{array}\right)\right) m ! S_{m}^{m}
\end{aligned}
$$

$$
\begin{aligned}
& \mathrm{d}_{1}=\sum_{\alpha=1}^{m}\left(\begin{array}{l}
n \\
\alpha
\end{array}\right) \alpha ! \quad S_{\alpha}^{m}-\mathrm{n} \\
& \left(\sum_{\beta=1}^{m}\left(\begin{array}{c}
n-1 \\
\beta
\end{array}\right) \beta ! S_{\beta}^{m}-(n-1)\right) \\
& =n^{m}-\mathbf{n}-\left((n-1)^{m}-(n-1)\right) \\
& =n^{m}-(n-1)^{m}-1
\end{aligned}
$$

To find $d_{r}$, the degree of any vertex in a subgraph in plane $P_{r}$ we have two subcases :

Subcase ( i ) : $\mathbf{n}-\mathbf{r}<\mathbf{m}$, here

$$
\begin{aligned}
& \mathbf{d}_{\mathrm{r}}=\mathbf{r}-1+\left(\left(\begin{array}{l}
\mathbf{n} \\
2
\end{array}\right)-\left(\begin{array}{c}
\mathbf{n}-\mathbf{r} \\
2
\end{array}\right) \quad .2\right. \text { ! } \\
& S_{2}^{m}+\left(\left(\begin{array}{c}
\mathbf{n} \\
3
\end{array}\right)-\left(\begin{array}{c}
\mathbf{n}-\mathbf{r} \\
3
\end{array}\right)\right) .3 ! S_{3}^{m}+\ldots \\
& +\left(\left(\begin{array}{c}
\mathbf{n} \\
\mathbf{n}-\mathbf{r}
\end{array}\right)-\left(\begin{array}{l}
\mathbf{n}-\mathbf{r} \\
\mathbf{n}-\mathbf{r}
\end{array}\right)\right) \cdot(\boldsymbol{n}-\boldsymbol{r}) ! \boldsymbol{S}_{\boldsymbol{n}-\boldsymbol{r}}^{\mathbf{m}} \\
& +\quad\left(\begin{array}{c}
\mathbf{n} \\
\mathbf{n}-\mathbf{r}+\mathbf{1}
\end{array}\right)(n-r+1) \text { ! } \\
& S_{n-r+1}^{m}+\ldots+\left(\begin{array}{c}
n \\
m
\end{array}\right) m ! S_{m}^{m} \\
& =r-1+\sum_{\alpha=1}^{m}\left(\begin{array}{l}
n \\
\alpha
\end{array}\right) \alpha ! \quad S_{\alpha}^{m}-\mathbf{n}- \\
& \left(\sum_{\beta=1}^{n-r}\left(\begin{array}{c}
n-r \\
\beta
\end{array}\right) \beta ! S_{\beta}^{m}-(n-r)\right) \\
& =r-1+n^{m}-\mathbf{n}-\left((n-r)^{m}-(n-\right. \\
& \boldsymbol{r})) \\
& =n^{m}-(n-r)^{m}-1
\end{aligned}
$$
as in case 1 )

Subcase ( ii ) : $\mathbf{n}-\mathbf{r} \geq \mathbf{m}$

$$
\begin{aligned}
& \left.\mathrm{d}_{\mathrm{r}}=\mathbf{r}-1+\left(\begin{array}{c}
\mathbf{n} \\
2
\end{array}\right)-\left(\begin{array}{c}
\mathbf{n}-\mathbf{r} \\
2
\end{array}\right)\right) .2 ! \\
& S_{2}^{m}+\left(\left(\begin{array}{l}
\mathbf{n} \\
3
\end{array}\right)-\left(\begin{array}{c}
\mathbf{n}-\mathbf{r} \\
3
\end{array}\right)\right) .3 ! S_{3}^{m}+\ldots \\
& \quad+\left(\left(\begin{array}{c}
\mathbf{n} \\
\mathbf{m}
\end{array}\right)-\left(\begin{array}{c}
\mathbf{n}-\mathbf{r} \\
\mathbf{m}
\end{array}\right)\right) m ! S_{m}^{m} \\
& \quad=r-1+\sum_{\alpha=1}^{m}\left(\begin{array}{l}
n \\
\alpha
\end{array}\right) \alpha ! \quad S_{\alpha}^{m}-\mathbf{n}- \\
& \left(\sum_{\beta=1}^{m}\left(\begin{array}{c}
n-r \\
\beta
\end{array}\right) \beta ! S_{\beta}^{m}-(\mathrm{n}-\mathbf{r})\right) \\
& \quad=n^{m}-(n-r)^{m}-1 \quad \text { (the same }
\end{aligned}
$$
as in case 1 )

Now, as before, $N_{r}$ is the number of vertices in plane $P_{r}$ which is given by :

$$
\mathbf{N}_{\mathbf{r}}=\left(\begin{array}{c}
\mathbf{n} \\
\mathbf{r}
\end{array}\right) \boldsymbol{r} ! \boldsymbol{S}_{\boldsymbol{r}}^{\boldsymbol{m}}
$$


hence the total number of edges of the graph is given by :

$$
\begin{aligned}
& |\mathrm{E}|=\frac{1}{2} \sum_{r=1}^{m} \mathrm{~N}_{\mathrm{r}} \cdot \mathrm{d}_{\mathrm{r}} \\
& =\frac{1}{2} \quad \sum_{r=1}^{m}\left(\begin{array}{l}
\mathbf{n} \\
r
\end{array}\right) r ! S_{r}^{m}\left(\left(n^{m}-1\right)-\right. \\
& \left.\left.(\boldsymbol{n}-\boldsymbol{r})^{\boldsymbol{m}}\right)\right) \\
& |\mathrm{E}|=\frac{1}{2} \quad\left(n^{m}-1\right) \sum_{r=1}^{m}\left(\begin{array}{l}
\mathrm{n} \\
\mathrm{r}
\end{array}\right) r ! S_{r}^{m}- \\
& \frac{1}{2} \sum_{r=1}^{m}(n-r)^{m}\left(\begin{array}{l}
\mathbf{n} \\
\mathbf{r}
\end{array}\right) r ! S_{r}^{m} \\
& =\frac{1}{2} \quad n^{m}\left(n^{m}-1\right)- \\
& \frac{1}{2} \sum_{r=1}^{m}\left(\begin{array}{l}
\mathbf{n} \\
\mathbf{r}
\end{array}\right)^{2} r ! S_{r}^{m}(n-r)^{m}, \quad \mathbf{m}<\mathbf{n}
\end{aligned}
$$

2.5 Example : $\mathrm{X}:=\left\{x_{1}, x_{2}, x_{3}, x_{4}, x_{5}\right\}$, $\mathrm{Y}:=\left\{y_{1}, y_{2}, y_{3}\right\}$

The number of vertices of the corresponding functional intersection graph $=3^{5}=243$

The number of edges $=\frac{1}{2} \times 243 \times 242$

$-\frac{1}{2} \sum_{r=1}^{3}(3-r)^{5}\left(\begin{array}{l}3 \\ r\end{array}\right) r ! S_{r}^{5}$

$$
\begin{gathered}
=29403 \\
\frac{1}{2}\left(32 \times 3 \times 1 ! S_{1}^{5}+3 \times 2 ! S_{2}^{5}+0\right),
\end{gathered}
$$

where $S_{1}^{5}=1$,

$$
\text { 2! } S_{2}^{5}=\sum_{s=1}^{2}(-1)^{2+s}\left(\begin{array}{l}
2 \\
s
\end{array}\right) s^{5}=-2
$$

$+2^{5}=30$,

hence $|E|=29403-\frac{1}{2}(96+90)$

$$
=\mathbf{2 9 3 1 0}
$$

2.6 Example : $\mathrm{X}:=\left\{x_{1}, x_{2}, x_{3}\right\}, \mathrm{Y}:=$ $\left\{y_{1}, y_{2}, y_{3}, y_{4}, y_{5}\right\}$

Number of vertices $=5^{3}=125$

$|\mathrm{E}|=$ number of edges $=\frac{1}{2} \times 125 \times 124$

$-\frac{1}{2} \sum_{r=1}^{3}\left(\begin{array}{l}5 \\ r\end{array}\right)(5-r)^{3} r ! S_{r}^{3}$,

where $S_{1}^{3}=S_{3}^{3}=1$

$$
S_{2}^{3}=\frac{1}{2 !} \sum_{s=1}^{2}(-1)^{s+2}\left(\begin{array}{l}
2 \\
s
\end{array}\right) s^{3}=3
$$

hence $|E|=\frac{1}{2} \times 125 \times 124-\frac{1}{2}(5 \times 64+10$ $\times 27 \times 6+10 \times 8 \times 6)$

$$
\begin{aligned}
& =7750-1210 \\
& =6540
\end{aligned}
$$

\section{References}

1. McKee, Terry A. and McMorris, F. R., Topics in intersection graph theory, (1999) SIAM.

2. Boyadzhiev, Khristo N., Clouse encounters with Stirling numbers of the second kind (2012) Mathematics magazine, 85 (4) pp 252-266. 
قمنا فى هذا البحث بحساب عدد الأحرف فى شكلين كل منهما عبارة عن رسم تقاطع ـ ففى

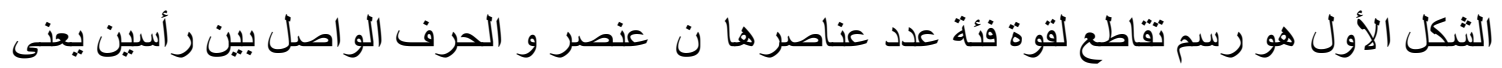

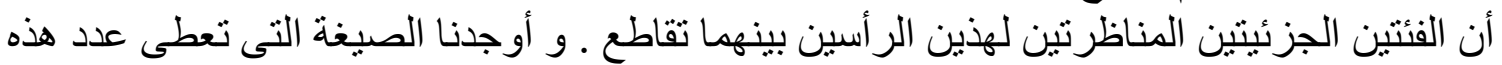

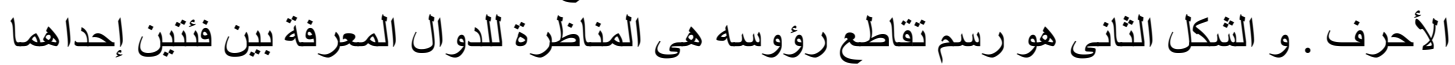

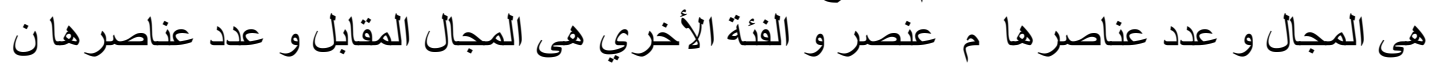

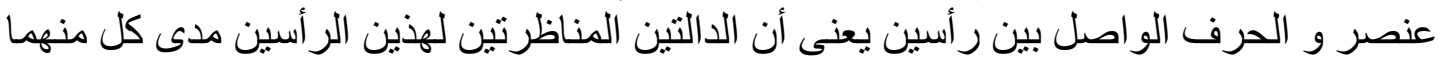

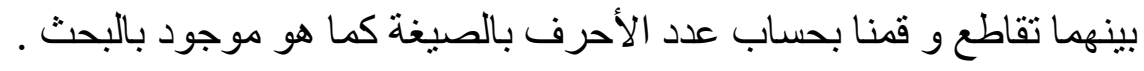


Delta J. Sci. 2016 Vol.37 